\title{
Metabolic syndrome associates with left atrial dysfunction
}

\author{
Nyman, K.
}

2018-07

Nyman , K, Granér , M , Pentikäinen, M O , Lundbom , J , Hakkarainen , A , Sirén , R , Nieminen, M S , Taskinen, M-R , Lundbom , N \& Lauerma , K 2018 , ' Metabolic syndrome associates with left atrial dysfunction ' , Nutrition, Metabolism and Cardiovascular Diseases, vol. 28 , no. 7 , pp. 727-734 . https://doi.org/10.1016/j.numecd.2018.02.008

http://hdl.handle.net/10138/299272

https://doi.org/10.1016/j.numecd.2018.02.008

cc_by_nc_nd

acceptedVersion

Downloaded from Helda, University of Helsinki institutional repository.

This is an electronic reprint of the original article.

This reprint may differ from the original in pagination and typographic detail.

Please cite the original version. 


\section{Accepted Manuscript}

Metabolic syndrome associates with left atrial dysfunction

K. Nyman, M. Granér, M.O. Pentikäinen, J. Lundbom, A. Hakkarainen, R. Sirén, M.S. Nieminen, M.-R. Taskinen, N. Lundbom, K. Lauerma

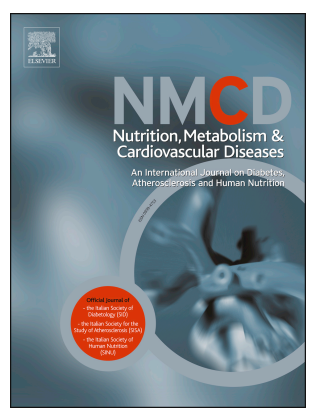

PII:

S0939-4753(18)30059-0

DOI:

10.1016/j.numecd.2018.02.008

Reference: NUMECD 1855

To appear in: Nutrition, Metabolism and Cardiovascular Diseases

Received Date: 6 October 2017

Revised Date: 5 February 2018

Accepted Date: 13 February 2018

Please cite this article as: Nyman K, Granér M, Pentikäinen M, Lundbom J, Hakkarainen A, Sirén R, Nieminen M, Taskinen M-R, Lundbom N, Lauerma K, Metabolic syndrome associates with left atrial dysfunction, Nutrition, Metabolism and Cardiovascular Diseases (2018), doi: 10.1016/ j.numecd.2018.02.008.

This is a PDF file of an unedited manuscript that has been accepted for publication. As a service to our customers we are providing this early version of the manuscript. The manuscript will undergo copyediting, typesetting, and review of the resulting proof before it is published in its final form. Please note that during the production process errors may be discovered which could affect the content, and all legal disclaimers that apply to the journal pertain. 
Metabolic syndrome associates with left atrial dysfunction

Nyman $K^{a}$, Granér $M^{b, c}$, Pentikäinen $M O^{b}$, Lundbom $J^{a}$, Hakkarainen $A^{a}$, Sirén $R^{d}$, Nieminen $M S^{b}$, Taskinen $\mathrm{M}-\mathrm{R}^{\mathrm{c}}$, Lundbom $\mathrm{N}^{\mathrm{a}}$, Lauerma $\mathrm{K}^{\mathrm{a}}$

${ }^{a}$ HUS Medical Imaging Center, Radiology, University of Helsinki and Helsinki University Hospital, Helsinki, Finland

${ }^{\mathrm{b}}$ Heart and Lung Center, Cardiology, Helsinki University Hospital, Helsinki, Finland

'Diabetes and Obesity Research Program, Research Programs' Unit, University of Helsinki and Clinical Research Institute, HUCH Ltd, Helsinki, Finland

${ }^{\mathrm{d} D e p a r t m e n t}$ of General Practice and Primary Health Care, Health Care Centre of City of Helsinki and University of Helsinki, Helsinki, Finland

Corresponding author: Dr. Kristofer Nyman, HUS Medical Imaging Center, Helsinki University

Hospital, P.O. Box 340, Haartmaninkatu 4, Helsinki, FI-00029 HUS, Finland; tel. +358 50427 1226;

fax +358 94717 4404; e-mail: kristofer.nyman@hus.fi.

Word counts:

Abstract: 230

Total (excl. abbreviations, references, declarations and tables): 4436

Number of references: 34

Figures: 1

Tables: 4 


\section{List of abbreviations}

$3 \mathrm{ch}=$ three-chamber

$\mathrm{AF}=$ atrial fibrillation

ATP $=$ adenosine triphosphate

$\mathrm{BMI}=$ body mass index

$\mathrm{BSA}=$ body surface area

$\mathrm{CMR}=$ cardiovascular magnetic resonance

$\mathrm{EDV}=$ end diastolic volume

$E F=$ ejection fraction

$\mathrm{ESV}=$ end systolic volume

${ }^{1} \mathrm{H}-\mathrm{MRS}={ }^{1} \mathrm{H}$-magnetic resonance spectroscopy

$\mathrm{HF}=$ heart failure

$\mathrm{HDL}=$ high-density lipoprotein cholesterol

HOMA-IR = homeostasis model assessment of insulin resistance

Hs-CRP = high-sensitivity $\mathrm{C}$-reactive protein

$\mathrm{LA}=$ left atrium

$\mathrm{LV}=$ left ventricle

MetS $=$ metabolic syndrome

$\mathrm{MRI}$ = magnetic resonance imaging

$\mathrm{SA}=$ short axis 
SAT = subcutaneous adipose tissue

T2DM = type 2 diabetes mellitus

$\mathrm{TG}=$ triglyceride

VAT $=$ visceral adipose tissue 
Abstract

Background and aims

Obesity and metabolic syndrome (MetS) are risk factors of atrial fibrillation (AF), but limited data exist on their effect on left atrial (LA) function. The aim of the study was to evaluate the effects of cardiac, hepatic and intra-abdominal ectopic fat depots and cardiometabolic risk factors on LA function in non-diabetic male subjects.

Methods and results

Myocardial and hepatic triglyceride contents were measured with $1.5 \mathrm{~T}{ }^{1} \mathrm{H}$-magnetic resonance spectroscopy and LA and left ventricular function, visceral adipose tissue (VAT), subcutaneous adipose tissue (SAT), epicardial and pericardial fat by magnetic resonance imaging (MRI) in 33 men with MetS and 40 men without MetS. LA volumes were assessed using a novel three-chamber orientation based MRI approach. LA ejection fraction (EF) was lower in MetS patients than in the control group (44 $\pm 7.7 \%$ in MetS vs. $49 \pm 8.6 \%$ in controls, $p=0.013$ ) without LA enlargement, indicating LA dysfunction. LA EF correlated negatively with waist circumference, body mass index, SAT, VAT, fasting serum insulin, and homeostasis model assessment of insulin resistance index, and positively with fasting serum high-density lipoprotein cholesterol. VAT was the best predictor of reduced LA EF.

\section{Conclusions}

MetS associates with subclinical LA dysfunction. Multiple components of MetS are related to LA dysfunction, notably visceral obesity and insulin resistance. Further studies are needed to elucidate the role of mechanical atrial remodeling in the development of AF.

\section{Keywords}


Cardiovascular magnetic resonance, proton magnetic resonance spectroscopy, metabolic syndrome, obesity, left atrial, ejection fraction, atrial remodeling, visceral adipose tissue, cardiac steatosis.

\section{Background}

Overweight and obesity are major global health concerns affecting around 2 billion people worldwide [1]. Central obesity is particularly hazardous to health associating with metabolic syndrome (MetS) and type 2 diabetes mellitus (T2DM). In MetS, lipid overflow will ultimately lead to fat accumulation not only around the viscera but also to non-adipose tissue such as liver, pancreas, skeletal muscle, and heart [2]. MetS increases the risk for morbidity and mortality from cardiovascular diseases including heart failure (HF) $[3,4]$. Left ventricular (LV) diastolic dysfunction with preserved systolic function is an early precursor of obesity cardiomyopathy leading eventually to HF. LV diastolic dysfunction has been associated with MetS and it has been shown to correlate with ectopic fat accumulation such as increased amount of pericardial or epicardial fat or hepatic fat content $[5,6]$.

Atrial fibrillation (AF) is the most common cardiac rhythm disorder and is associated with significant morbidity and mortality, especially that arising from stroke and HF, imposing a public health burden $[7,8]$. The prevalence of AF increases with age from less than $0.16 \%$ in people younger than 49 years to over $10 \%$ in persons aged over 80 years [7]. AF is commonly associated with overweight and obesity while other major risk factors include male sex, hypertension, HF, coronary artery disease, diabetes, sleep apnea, and excessive alcohol use [8]. 
In obese patients without $A F$, left atrial (LA) enlargement has been related to hypertension and LV dysfunction [9]. However, magnetic resonance imaging (MRI) data regarding LA function in MetS is limited. Atrial remodeling is defined as a spectrum of pathophysiological changes in atrial structure and function that occur in response to stresses imposed by conditions such as hypertension, HF, T2DM, and obesity [10]. Atrial remodeling may be subdivided to structural remodeling, as measured by LA size, and mechanical remodeling, as measured by LA function. Milder, often subclinical forms of atrial dysfunction may show decreased LA ejection fraction (EF) without LA enlargement.

The aim of the study was to assess LA function in men with MetS without known heart diseases and to examine the association of different ectopic fat depots and cardiometabolic risk factors with LA dysfunction.

\section{Methods}

\section{Study design}

Study population consisted of 73 men from the same study cohort as previously described [5]. Subjects were allocated into two groups (MetS present and MetS absent), based on the following criteria: 1) waist circumference $\geq 94 \mathrm{~cm}$, and 2) having $\geq 2$ abnormal findings as per the harmonized definition of MetS [11]. Thirty-three participants fulfilled the criteria for MetS. In these participants, myocardial ischemia was excluded by means of adenosine stress perfusion MR. Exclusion criteria and medications are described in the supplemental material.

The study was approved by the Ethics Committee of the Department of Medicine, Hospital District of Helsinki and Uusimaa, and each subject provided written informed consent. 
Body mass index (BMI), waist circumference, blood pressure measurements and blood sample examination and analysis were performed as previously described [12].

\section{CMR protocol and analysis}

Cardiac MR images were acquired using 1.5T imager (Magnetom Avanto; Siemens, Erlangen, Germany) and analysis was performed by two radiologists with experience of CMR. Imaging parameters are provided in the supplement.

Three-chamber (3ch) oriented images were used for measuring LA area in a single plane (Figure 1 in supplemental material). All phases of the cine images were inspected and minimum and maximum LA area were planimetered using a diagnostic radiologic workstation (Impax 6 software, Agfa Healthcare, Mortsel, Belgium), and reported as LA 2D model. LA area measured in 3ch MR image is a reliable indicator of LA size [13] but volumetric CMR data based on 3ch images has not been reported. For 3D model of the LA we used prolate ellipsoid model, which is commonly used for estimating LA volume in echocardiographic studies. Volume of the ellipsoid was calculated according to formula: $V=$ $\frac{4}{3} \pi a b c$, where $\pi a b=3 \mathrm{chA}$, area measured in the $3 c h$ image, and $c=0.5^{*} h$, where $h$ equals maximum craniocaudal diameter of the LA in a short axis (SA)-oriented image at the midline of the LA (Figure 2 in supplemental material). Both measurements were done in end-systolic and end-diastolic images to obtain minimum and maximum LA volumes, and to calculate EF. Volume parameters are reported also as indexed to the subject's body surface area (BSA).

Comparison of LA assessment of 3ch prolate ellipsoid model and traditional Simpson's method, considered as gold standard, was performed for a group $(n=10)$ of study subjects with sufficient data available for both methods. In these subjects, LA volume was 
planimetered from a stack of SA cine images with Simpson's method using dedicated software (QMass MR v.7.6, Medis Medical Imaging Systems, Leiden, Netherlands).

Quantification of cardiac, hepatic and abdominal fat depots by MRI and ${ }^{1} \mathrm{H}-\mathrm{MRS}$

For measuring myocardial and hepatic triglyceride (TG) content, ${ }^{1} \mathrm{H}$-magnetic resonance spectroscopy $\left({ }^{1} \mathrm{H}-\mathrm{MRS}\right)$ was performed in a $1.5 \mathrm{~T}$ MR imager (Magnetom Avanto; Siemens). MRS protocol is presented in the supplement. Distributions of visceral and subcutaneous adipose tissue (VAT and SAT) and epicardial and pericardial fat were measured as previously described $[5,12]$.

\section{Statistical analyses}

All statistical analyses were performed with IBM SPSS Statistics for Windows, version 23.0 (IBM Corp., Armonk, NY, USA). Normality of continuous variables was analyzed by the Kolmogorov-Smirnov test. Logarithmic, square root or reciprocal transformation of variables was performed, if necessary. Data are presented as frequencies or percentages for categorical variables, as means \pm SD for normally distributed continuous variables, and as medians (range) for skewed variables. Between-group differences were assessed by the Mann-Whitney U test, unpaired t-test, or the chi-square test, as appropriate. Depending on distribution of values, Pearson or Spearman correlation coefficients were used to assess the relationship between LA parameters and clinical parameters and results of ectopic fat quantification. Univariable linear regression model was used with and without ageadjustment to examine the possible factors of atrial dysfunction using LA EF as dependent variable. Multivariable stepwise regression analyses were performed to determine the independent predictors of LA EF. Three-chamber-based LA volume assessment and SA-based 
Simpson's method were compared using Pearson correlation and Bland-Altman analysis. For all analyses, $p<0.05$ was considered statistically significant.

\section{Results}

Clinical and biochemical characteristics, body fat distribution and CMR data

Demographic differences between groups (Table 1) have been reported earlier in detail [5]. Briefly, subjects with MetS were older, had larger waist circumference, and higher BMI and blood pressure than subjects without MetS. All ectopic fat depots were larger in subjects with MetS than in controls. More unfavorable plasma lipid profile and markers of glucose metabolism were noted in participants with MetS as compared to controls.

LA CMR data is outlined in Table 1. BSA-indexed LA areas (2D model) or volumes (3D model) did not differ between groups. In 2D and 3D models, LA EF was higher in the group without MetS than in the MetS group. LV data (Table 1 in supplemental material) have been reported earlier [5]. 
Table 1. Clinical and biochemical characteristics, fat compartments and left atrial size and function.

\begin{tabular}{|c|c|c|c|}
\hline & MetS present & Mets absent & $\mathbf{p}$ \\
\hline $\mathbf{N}$ & 33 & 40 & \\
\hline Age (years) & $48.6 \pm 6.8$ & $40.4 \pm 7.6$ & $<0.001$ \\
\hline Body mass index $\left(\mathrm{kg} / \mathrm{m}^{2}\right)$ & $30.1 \pm 4.7$ & $24.1 \pm 2.2$ & $<0.001$ \\
\hline Waist circumference $(\mathrm{cm})$ & $107(94-127)$ & $88(71-93.5)$ & $<0.001$ \\
\hline Current smokers (N, \%) & $10(32)$ & $6(15)$ & 0.075 \\
\hline Heart rate $(1 / \mathrm{min})$ & $69 \pm 13$ & $62 \pm 8.8$ & 0.006 \\
\hline Systolic blood pressure (mmHg) & $132 \pm 14$ & $115 \pm 9.9$ & $<0.001$ \\
\hline Diastolic blood pressure (mmHg) & $88 \pm 9.9$ & $74 \pm 6.1$ & $<0.001$ \\
\hline Total cholesterol (mmol/l) & $5.1 \pm 0.85$ & $4.4 \pm 0.80$ & 0.001 \\
\hline High-density lipoprotein cholesterol (mmol/l) & $1.1 \pm 0.25$ & $1.5 \pm 0.39$ & $<0.001$ \\
\hline Low-density lipoprotein cholesterol (mmol/l) & $3.2 \pm 0.79$ & $2.5 \pm 0.67$ & 0.001 \\
\hline Triglycerides (mmol/l) & $2.0 \pm 1.0$ & $0.83 \pm 0.31$ & $<0.001$ \\
\hline fP-ALT (U/I) & $34(16-260)$ & $22(7-59)$ & $<0.001$ \\
\hline fP-glucose ( $\mathrm{mmol} / \mathrm{l})$ & $5.8 \pm 0.58$ & $5.1 \pm 0.40$ & $<0.001$ \\
\hline fS-insulin (mU/I) & $9.2(3.3-28)$ & $3.0(0.9-9.7)$ & $<0.001$ \\
\hline HOMA-IR index & $2.2(0.75-6.8)$ & $0.6(0.2-2.2)$ & $<0.001$ \\
\hline Hs-CRP (mg/l) & $0.97(0.03-12)$ & $0.33(0-5.8)$ & $<0.001$ \\
\hline NT-proBNP (ng/l) & $27(5-151)$ & $23(5-74)$ & 0.210 \\
\hline Myocardial triglyceride content (\%) & $0.970(0.31-4.76)$ & $0.450(0.14-1.39)$ & $<0.001$ \\
\hline Epicardial fat $\left(\mathrm{mm}^{2}\right)$ & $895(385-2020)$ & $542(251-1130)$ & $<0.001$ \\
\hline Pericardial fat $\left(\mathrm{mm}^{2}\right)$ & $1990(750-6130)$ & $611(66-1580)$ & $<0.001$ \\
\hline Hepatic triglyceride content (\%) & $6.04(1.30-31.7)$ & $0.880(0.27-6.16)$ & $<0.001$ \\
\hline SAT $\left(\mathrm{cm}^{3}\right)$ & $4710 \pm 1650$ & $2050 \pm 839$ & $<0.001$ \\
\hline $\operatorname{VAT}\left(\mathrm{cm}^{3}\right)$ & $3380(1260-5740)$ & $883(67-3170)$ & $<0.001$ \\
\hline LA 3ch Area EF (\%) & $32.1 \pm 6.3$ & $36.8 \pm 8.4$ & 0.008 \\
\hline LA 3ch Area ED $\left(\mathrm{mm}^{2}\right)$ & $2230 \pm 470$ & $1980 \pm 320$ & 0.012 \\
\hline LA 3ch Area ED/BSA $\left(10^{-4}\right)$ & $10.2 \pm 2.0$ & $10.1 \pm 1.6$ & 0.786 \\
\hline LA 3ch Area ES $\left(\mathrm{mm}^{2}\right)$ & $1520 \pm 350$ & $1250 \pm 270$ & 0.001 \\
\hline LA 3ch Area ES/BSA $\left(10^{-4}\right)$ & $6.9 \pm 1.5$ & $6.3 \pm 1.3$ & 0.095 \\
\hline LA EF (\%) & $44 \pm 7.7$ & $49 \pm 8.6$ & 0.013 \\
\hline LA EDV (ml) & $86 \pm 23$ & $79 \pm 17$ & 0.160 \\
\hline LA EDV/BSA $\left(\mathrm{ml} / \mathrm{m}^{2}\right)$ & $39 \pm 9.7$ & $40 \pm 8.5$ & 0.638 \\
\hline LA ESV (ml) & $48 \pm 15$ & $40 \pm 11$ & 0.015 \\
\hline LA ESV/BSA $\left(\mathrm{ml} / \mathrm{m}^{2}\right)$ & $22 \pm 6.5$ & $20 \pm 5.1$ & 0.285 \\
\hline
\end{tabular}

Data are expressed as means $( \pm \mathrm{SD})$, medians (range) or as frequencies (\%). Abbreviations: $\mathrm{fP}$, fasting plasma; fS, fasting serum; hs-CRP, high-sensitivity C-reactive protein; HOMA-IR, homeostasis model assessment of insulin resistance; NT-proBNP, N-terminal pro-brain natriuretic peptide; SAT, 
subcutaneous adipose tissue; VAT, visceral adipose tissue; LA, left atrial; EF, ejection fraction; ED, end diastolic; ES, end systolic; BSA, body surface area.

Correlation analyses of clinical and biochemical parameters and body fat depots with LA size and function

In both 2D and 3D models of LA EF, age, waist circumference, BMI, fasting plasma glucose, fasting serum insulin, homeostasis model assessment of insulin resistance (HOMA-IR) index, high-sensitivity C-reactive protein (hs-CRP), hepatic TG content, VAT, and SAT correlated inversely with LA EF and high-density lipoprotein cholesterol (HDL) correlated positively with LA EF (Table 2). Scatter plots demonstrating the correlations of LA EF (3D model) with selected contributing factors of MetS are shown in Figure 1. Negative correlation of myocardial TG content, epicardial and pericardial fat with LA EF was noted in the 2D LA model, but these findings did not remain significant in the 3D LA model. Correlations between clinical and biochemical parameters and fat compartments and LA volumes are provided in the supplement (Table 2 in supplemental material).

Univariate linear regression analysis was performed for the 3D model of LA EF (Table 3). In age-adjusted linear regression model, regression coefficients with LA EF and waist circumference, BMI, SAT, VAT, fasting serum insulin, HOMA-IR index, and HDL were significant. Stepwise multivariable regression analysis was performed to further examine the relationship of ectopic fat depots and LA EF (Table 4). It revealed that VAT followed by SAT were the best predictors of reduced LA EF when age, BMI, and hepatic TG content were taken into account. 
Table 2. Correlations between clinical and biochemical parameters and fat compartments and LA function.

\begin{tabular}{lcccc}
\hline Variable & LA EF (\%) & 2D model & \multicolumn{2}{c}{ LA EF (\%) 3D model } \\
\hline & $\beta$ & $p$ & $\beta$ & $p$ \\
\hline Age & -0.249 & $0.034{ }^{\dagger}$ & -0.260 & $0.026^{\dagger}$ \\
\hline Body mass index & -0.372 & $0.001^{*}$ & -0.270 & $0.021^{\dagger}$ \\
\hline Waist circumference & -0.420 & $<0.001^{*}$ & -0.349 & $0.002^{*}$ \\
\hline Systolic blood pressure & -0.103 & 0.386 & -0.121 & 0.309 \\
\hline Diastolic blood pressure & -0.117 & 0.325 & -0.146 & 0.216 \\
\hline & & & & \\
\hline SELECTED BIOCHEMICAL PARAMETERS & & & & \\
\hline High-density lipoprotein cholesterol & 0.333 & $0.004^{*}$ & 0.316 & $0.006^{*}$ \\
\hline Low-density lipoprotein cholesterol & -0.022 & 0.856 & -0.091 & 0.442 \\
\hline fP-glucose (mmol/l) & -0.314 & $0.007^{*}$ & -0.269 & $0.021^{\dagger}$ \\
\hline fS-insulin (mU/I) & -0.433 & $<0.001^{*}$ & -0.371 & $0.001^{*}$ \\
\hline HOMA-IR index & -0.439 & $<0.001^{*}$ & -0.373 & $0.001^{*}$ \\
\hline Hs-CRP (mg/l) & -0.361 & $0.002^{*}$ & -0.281 & $0.018^{\dagger}$ \\
\hline NT-proBNP (ng/l) & -0.158 & 0.181 & -0.112 & 0.344 \\
\hline & & & & \\
\hline ECTOPIC FAT DEPOTS & & & & \\
\hline Myocardial triglyceride content (\%) & -0.305 & $0.009^{*}$ & -0.165 & 0.166 \\
\hline Epicardial fat (mm ${ }^{2}$ ) & -0.260 & $0.026 \dagger$ & -0.124 & 0.294 \\
\hline Pericardial fat (mm ${ }^{2}$ ) & -0.269 & $0.021 \dagger$ & -0.199 & 0.092 \\
\hline SAT (mm ${ }^{2}$ ) & -0.415 & $0.001^{*}$ & -0.356 & $0.003^{*}$ \\
\hline VAT (mm ${ }^{2}$ ) & -0.450 & $<0.001^{*}$ & -0.352 & $0.003^{*}$ \\
\hline Hepatic triglyceride content (\%) & -0.370 & $<0.001^{*}$ & -0.314 & $0.007^{*}$ \\
\hline & & & & \\
\hline
\end{tabular}

Abbreviations: LA, left atrial; $E F$, ejection fraction; fP, fasting plasma; $f S$, fasting serum; Hs-CRP, highsensitivity C-reactive protein; HOMA-IR, homeostasis model assessment of insulin resistance; NTproBNP, N-terminal pro-brain natriuretic peptide; SAT, subcutaneous adipose tissue; VAT, visceral adipose tissue. ${ }^{*} p<0.01,+p<0.05$ 
Table 3. Results of univariate regression analysis.

\begin{tabular}{lcccc}
\hline Dependent variable: LA EF (3D model) & Beta & $\mathbf{R}^{2}$ & Adj. $\mathbf{R}^{2}$ & $\boldsymbol{p}$ \\
\hline Age & -0.260 & 0.068 & 0.055 & 0.026 \\
\hline Body mass index $\left(^{-1}\right.$ ) & 0.318 & 0.101 & 0.089 & $0.006^{*}$ \\
\hline Waist circumference $\left(^{-1}\right)$ & 0.359 & 0.129 & 0.117 & $0.002^{*}$ \\
\hline Systolic blood pressure & -0.121 & 0.015 & 0.001 & 0.309 \\
\hline Diastolic blood pressure & -0.146 & 0.021 & 0.008 & 0.216 \\
\hline High-density lipoprotein cholesterol & 0.316 & 0.100 & 0.087 & $0.006^{*}$ \\
\hline Low-density lipoprotein cholesterol & -0.091 & 0.008 & -0.006 & 0.442 \\
\hline fP-glucose (In) & -0.293 & 0.086 & 0.073 & 0.012 \\
\hline fS-insulin (In) & -0.362 & 0.131 & 0.119 & $0.002^{*}$ \\
\hline HOMA-IR index (In) & -0.371 & 0.138 & 0.125 & $0.001^{*}$ \\
\hline Hs-CRP $\left(^{-1 / 4}\right.$ ) & -0.294 & 0.086 & 0.073 & 0.013 \\
\hline NT-proBNP (In) & -0.165 & 0.027 & 0.014 & 0.163 \\
\hline Myocardial triglyceride content (In) & -0.170 & 0.029 & 0.015 & 0.153 \\
\hline Epicardial fat (In) & -0.137 & 0.019 & 0.005 & 0.247 \\
\hline Pericardial fat (In) & -0.171 & 0.029 & 0.015 & 0.149 \\
\hline Hepatic triglyceride content (In) & -0.267 & 0.071 & 0.058 & 0.024 \\
\hline SAT (In) & -0.320 & 0.103 & 0.089 & $0.007^{*}$ \\
\hline VAT (In) & -0.326 & 0.107 & 0.093 & $0.006^{*}$ \\
\hline
\end{tabular}

Abbreviations: $L A$, left atrial; $E F$, ejection fraction; $f P$, fasting plasma; $f S$, fasting serum; $H s-C R P$, highsensitivity C-reactive protein; HOMA-IR, homeostasis model assessment of insulin resistance; NTproBNP, N-terminal pro-brain natriuretic peptide; SAT, subcutaneous adipose tissue; VAT, visceral adipose tissue. ${ }^{*} p<0.05$ after adjusting for age 
Table 4. Results of stepwise multivariable regression analysis.

Dependent variable: LA EF (3D model)

\begin{tabular}{lcccccc}
\hline & \multicolumn{2}{c}{ Model 1 } & \multicolumn{2}{c}{ Model 2 } & \multicolumn{2}{c}{ Model 3 } \\
\cline { 2 - 7 } Age & $\beta$ & $\mathrm{p}$ & $\beta$ & $\mathrm{p}$ & $\beta$ & $\mathrm{p}$ \\
\cline { 2 - 7 } & -0.112 & 0.427 & -0.164 & 0.189 & -0.172 & 0.157 \\
Body mass index $\left(^{-1}\right)$ & 0.170 & 0.324 & 0.169 & 0.421 & 0.318 & $0.007^{*}$ \\
\hline Hepatic triglyceride content $(\mathbf{I n})$ & -0.007 & 0.973 & -0.041 & 0.826 & -0.067 & 0.698 \\
\hline SAT (In) & -0.150 & 0.524 & -0.320 & $0.007^{*}$ & --- & --- \\
\hline VAT (In) & -0.326 & $0.006^{*}$ & --- & --- & --- & --- \\
\hline & & & & & & \\
\hline Adjusted R & 0.093 & $0.006^{*}$ & 0.089 & $0.007^{*}$ & 0.088 & $0.007^{*}$ \\
\hline
\end{tabular}

Abbreviations: LA, left atrial; EF, ejection fraction; SAT, subcutaneous adipose tissue; VAT, visceral adipose tissue. ${ }^{*} p<0.01$

Comparison of LA volume assessment by 3ch prolate ellipsoid method and SA volumetric method

LA measurements obtained with 3ch-based volume assessment correlated highly $(r=0.97$ for end diastolic volume (EDV), $r=0.90$ for end systolic volume (ESV), and $r=0.93$ for $E F$, $p<0.001$ for all correlations) with volumetric SA-based Simpson's method. Bland-Altman analysis (Figure 3 in supplemental material) demonstrated mean difference of LA EDV -1.06 $\pm 3.60 \mathrm{ml}$, LA ESV $-1.54 \pm 3.12 \mathrm{ml}$ and EF $0.83 \pm 3.16 \%$.

\section{Discussion}

To our knowledge, this is the first study utilizing MRI to assess LA function, and to combine the data with MRI and ${ }^{1} \mathrm{H}-\mathrm{MRS}$ derived quantification of major ectopic fat depots including all three cardiac fat depots and liver, and a wide range of clinical parameters in subjects with MetS. We found that MetS is associated with subclinical LA dysfunction in male subjects free of cardiovascular disease. Cross-sectional correlation analysis revealed that LA dysfunction is 
associated with several contributory factors of MetS including waist circumference, VAT, SAT, insulin resistance, and dyslipidemia.

LA function is important for optimum cardiac performance in three distinct phases. 1) LA serves as a reservoir of pulmonary venous return during LV systole. 2) During LV early diastole, it serves as a conduit and its function is modulated by LV diastolic performance. 3) At the late phase of LV diastole, LA acts as a pump boosting LV filling. In HF, with sustained increases in LV and LA pressure, LA dilatation occurs and LA contractile reserve becomes exhausted. In end-stage HF, this leads to a change of LA to a passive conduit dictated by ventricular distensibility [14]. In AF, the pump function of LA is lost due to dyssynchronous contraction of myocytes.

We found that in MetS, LA function is decreased as measured by LA EF without the enlargement of LA. Notably, our study subjects were asymptomatic and excluded for known heart diseases, including coronary artery disease, AF, and (clinical) heart failure. The decrease in LA EF indicates the presence of LA mechanical remodeling, an entity with increasing interest in the recent literature $[10,15]$. When comparing LA measurements to patient outcomes, LA EF was found superior and incremental to LA volume with regard to the assessment of mortality risk in general population [16]. Lower LA EF has also been associated with a poorer prognosis in patients with HF [17] and non-ischemic cardiomyopathies [18]. LA remodeling can be reversible, especially at early stages, and may be treated with medications such as angiotensin-converting enzyme inhibitors or angiotensin II receptor antagonists [19]. Echocardiographic studies have also shown improved atrial function with weight loss [20]. 
Obesity and MetS are well-known risk factors of AF and decreased LA EF has been shown to increase the risk for AF independent of LA size $[17,21]$. Among other causes, functional LA remodeling has been suggested as a factor preceding AF. However, the pathophysiological mechanisms of atrial remodeling and development of AF in obesity are complex and remain elusive. They include unfavorable genotype and lifestyle risk factors such as sleep apnea, hypertension, hyperlipidemia, T2DM, alcohol, and smoking, impaired diastolic function causing atrial stretch, focal adiposity, and inflammation resulting in scar tissue [8]. Consequently, structural, functional and electrical changes occur forming the substrate for AF. Evaluating LA function with a relatively simple 3ch CMR method may hence provide valuable clinical information to find particularly those obese persons at risk for developing AF or other cardiovascular events.

In our study, obesity and visceral obesity in particular were associated with LA dysfunction. Interestingly, with the exception of blood pressure, all other components (waist circumference, insulin resistance and dyslipidemia (low HDL)) of MetS were related to LA dysfunction. Waist and VAT were better predictors of LA dysfunction than BMI thus emphasizing the role of visceral obesity in LA remodeling respectively as earlier reported in LV remodeling [5]. In a recent population-based study, LA enlargement was associated with visceral obesity, but LA function was not evaluated [22]. In our study, liver fat was also associated with lower LA EF, but correlation did not remain significant after adjusting for age.

Insulin resistance is a key component of MetS associated with several pathogenetic abnormalities that lead to HF and increase cardiovascular mortality [4]. In our non-diabetic study population, insulin resistance was associated with LA dysfunction as shown by inverse 
correlation of blood glucose and insulin levels, and HOMA-IR index with LA EF. Our finding is in line with an earlier MRI study reporting an association of T2DM and lowered LA EF [23]. Mechanisms to explain the role of obesity, insulin resistance or T2DM in cardiac dysfunction are diverse. Those include abnormalities in contractile proteins, impaired relaxation or contraction, impairment of glucose transport, influx of free fatty acids leading to increase of $\beta$-oxidation, oxidative stress, and lipotoxicity; impairment of microvascular circulation, and neurohormonal and sympathetic nervous system activation [4]. Neurohormonal abnormalities include imbalance of adipokine production, and overactivity of reninangiotensin-aldosterone and endocannabinoid system. Adipokines are bioactive substances produced by adipose tissue and they largely function as modulators of inflammation [24]. Visceral obesity in particular leads to the upregulation of proinflammatory adipokines and the downregulation of anti-inflammatory adipokines which contribute to the pathogenesis of cardiovascular diseases. Additionally, renin-angiotensin system is contributor to and target of many components of MetS [4]. At the cardiac level increased angiotensin 2 activity promotes oxidative stress, and at the vascular level angiotensin 2 overproduction from hypertrophic adipocytes in obesity contribute to hypertension.

In our study population, cardiac steatosis was not a key factor of LA dysfunction although association of myocardial TG content with reduced LA EF in 2D model was noted. To our knowledge, there are no earlier ${ }^{1} \mathrm{H}$-MRS studies where myocardial TG content has been examined in atrial dysfunction or AF. In contrast to other main ectopic fat depots, intramyocytic TG is not a stable deposit of adipose tissue, but a relatively rapidly changing pool of lipids where up to three or four-fold increase has been reported following $48-72 \mathrm{~h}$ fasting in lean subjects [25]. In the ventricles, normal heart utilizes fatty acids and glucose as main energy sources with a ratio of 3:1 [26]. In the setting of obesity, the balance is shifted 
more towards consumption of fatty acids due to increased $\beta$-oxidation [27]. Under aerobic conditions, fatty acid oxidation yields more adenosine triphosphate (ATP) per gram of substrate but takes $10-15 \%$ more $\mathrm{O}_{2}$ per amount of ATP than glucose [28]. During chronic hypoxia and heart failure, ventricular metabolism shifts from fatty acid to glucose utilization. For the atria, substrate preference has not been investigated but it may not differ from that in the ventricles [28]. Analogously, whether the measurement of the myocardial TG content from the ventricular septal wall corresponds to the TG content of the LA myocardium is unclear. Theroretically, myocardial TG content may act ambiguously in obesity-related subclinical atrial dysfunction, as it may increase due to accelerated free fatty acid uptake and $\beta$-oxidation, and decrease in response to the higher energy demand, oxidative stress, and increased glycolysis. In conclusion, the dynamical role of intramyocytic lipids will merit further studies.

\section{Limitations}

Study population was limited to men to exclude the effects of hormonal variability. As a potential source of bias, MetS subjects were older than controls. According to previous cross-sectional imaging studies in normal population, data concerning LA remodeling in aging is inconsistent. Earlier studies showed no significant influence of age on LA volume and function [29-31] but in recent study by Maceira et al. [32], a mild age influence on LA function was found. In our control population of 40 men, age did not correlate with LA size or function, but we still performed an age-adjustment for the linear regression analyses. Another source of bias is that the measurement of LA volume did not cover the whole volume of LA but was based on a single image plane of 3ch images with the third dimension taken into account by measuring the LA diameter in perpendicular SA plane. Absolute LA 
volumes correlated well with those measured from SA images with Simpson's method considered as gold standard in our small study population, but a larger patient cohort incorporating a variety of atrial disease states will be needed to validate our novel method of deriving LA volumes from three-chamber oriented CMR images. Nevertheless, the absolute LA volume values we obtained were comparable to earlier CMR and multidetector computed tomography studies calculated with Simpson's method [31, 33, 34]. Phasic LA function analysis could have been informative to further evaluate the mechanism of atrial dysfunction and remodeling. Due to relatively small sample size high interrelationship of components of MetS limited the assessment of independent predictors of LA remodeling. Finally, due to cross-sectional study design, causal inferences are limited.

\section{Conclusions}

MetS associates with subclinical LA dysfunction as demonstrated by decreased LA EF without the enlargement of LA. According to previous knowledge, this represents LA mechanical remodeling that may result to incident of $\mathrm{AF}, \mathrm{HF}$ or mortality from cardiovascular disease. We found that multiple co-factors of MetS are related to LA dysfunction, notably visceral obesity and insulin resistance. The role of cardiac steatosis in LA dysfunction was not essential and remains to be clarified. Due to the heterogeneous phenotype of MetS patients and multi-factorial mechanisms, risk stratification for obesity cardiomyopathy or HF for individual person remains unclear and merits further studies. In the clinical context, early recognition of obese patients with adverse atrial remodeling may help to focus intervention and improve patient outcome.

\section{Acknowledgements}


We thank Hannele Hildén, Virve Naatti, Helinä Perttunen-Nio, and Sirpa Stick for skillful laboratory work; and Marja Piitulainen, Miika Paukkunen, and Laura Impivaara for excellent technical assistance.

Declarations

Competing interests

None.

\section{Funding}

This study was supported by grants from Helsinki University Hospital Research Foundation (grants TLD8100096 and TYH2009235), Finnish Foundation for Cardiovascular Research, Instrumentarium Science Foundation, Finnish Cultural Foundation, Finnish Medical Foundation, and Orion Research Foundation.

\section{Availability of data}

The data that support the findings of this study are available on request from the corresponding author $[\mathrm{KN}]$ on reasonable request. The data are not publicly available due to them containing information that could compromise research participant privacy/consent. 


\section{References}

1. WHO Fact Sheet: Obesity and overweight. Updated June 2016 [http://www.who.int/mediacentre/factsheets/fs311/en/]

2. Despres JP: Body fat distribution and risk of cardiovascular disease: an update. Circulation 2012, 126(10):1301-1313.

3. Mottillo S, Filion KB, Genest J, Joseph L, Pilote L, Poirier P, Rinfret S, Schiffrin EL, Eisenberg MJ: The metabolic syndrome and cardiovascular risk a systematic review and metaanalysis. J Am Coll Cardiol 2010, 56(14):1113-1132.

4. Perrone-Filardi P, Paolillo S, Costanzo P, Savarese G, Trimarco B, Bonow RO: The role of metabolic syndrome in heart failure. Eur Heart J 2015, 36(39):2630-2634.

5. Nyman K, Graner M, Pentikainen MO, Lundbom J, Hakkarainen A, Siren R, Nieminen MS, Taskinen MR, Lundbom N, Lauerma K: Cardiac steatosis and left ventricular function in men with metabolic syndrome. J Cardiovasc Magn Reson 2013, 15:103-429X-15-103.

6. Graner M, Nyman K, Siren R, Pentikainen MO, Lundbom J, Hakkarainen A, Lauerma K, Lundbom N, Nieminen MS, Taskinen MR: Ectopic fat depots and left ventricular function in nondiabetic men with nonalcoholic fatty liver disease. Circ Cardiovasc Imaging 2014, 8(1):10.1161/CIRCIMAGING.114.001979. Print 2015 Jan.

7. Zoni-Berisso M, Lercari F, Carazza T, Domenicucci S: Epidemiology of atrial fibrillation: European perspective. Clin Epidemiol 2014, 6:213-220.

8. Nalliah CJ, Sanders P, Kottkamp H, Kalman JM: The role of obesity in atrial fibrillation. Eur Heart J 2016, 37(20):1565-1572.

9. Alpert MA, Omran J, Mehra A, Ardhanari S: Impact of obesity and weight loss on cardiac performance and morphology in adults. Prog Cardiovasc Dis 2014, 56(4):391-400.

10. Hoit BD: Left Atrial Remodeling: More Than Just Left Atrial Enlargement. Circ Cardiovasc Imaging 2017, 10(2):10.1161/CIRCIMAGING.117.006036.

11. Alberti KG, Eckel RH, Grundy SM, Zimmet PZ, Cleeman JI, Donato KA, Fruchart JC, James WP, Loria CM, Smith SC,Jr, International Diabetes Federation Task Force on Epidemiology and Prevention, Hational Heart, Lung, and Blood Institute, American Heart Association, World Heart Federation, International Atherosclerosis Society, International Association for the Study of Obesity: Harmonizing the metabolic syndrome: a joint interim statement of the International Diabetes Federation Task Force on Epidemiology and Prevention; National Heart, Lung, and Blood Institute; American Heart Association; World Heart Federation; International Atherosclerosis Society; and International Association for the Study of Obesity. Circulation 2009, 120(16):1640-1645. 
12. Graner M, Siren R, Nyman K, Lundbom J, Hakkarainen A, Pentikainen MO, Lauerma K, Lundbom N, Adiels M, Nieminen MS, Taskinen MR: Cardiac steatosis associates with visceral obesity in nondiabetic obese men. J Clin Endocrinol Metab 2013, 98(3):1189-1197.

13. Maceira AM, Cosin-Sales J, Roughton M, Prasad SK, Pennell DJ: Reference left atrial dimensions and volumes by steady state free precession cardiovascular magnetic resonance. J Cardiovasc Magn Reson 2010, 12:65-429X-12-65.

14. Prioli A, Marino $P$, Lanzoni $L$, Zardini $P$ : Increasing degrees of left ventricular filling impairment modulate left atrial function in humans. Am J Cardiol 1998, 82(6):756-761.

15. Wijesurendra RS, Rider OJ, Neubauer S: Left Atrial Volumes in Health and Disease Measured Using Cardiac Magnetic Resonance. Circ Cardiovasc Imaging 2017, 10(2):10.1161/CIRCIMAGING.117.006124.

16. Gupta S, Matulevicius SA, Ayers CR, Berry JD, Patel PC, Markham DW, Levine BD, Chin $\mathrm{KM}$, de Lemos JA, Peshock RM, Drazner MH: Left atrial structure and function and clinical outcomes in the general population. Eur Heart J 2013, 34(4):278-285.

17. Pellicori P, Zhang J, Lukaschuk E, Joseph AC, Bourantas CV, Loh H, Bragadeesh T, Clark AL, Cleland JG: Left atrial function measured by cardiac magnetic resonance imaging in patients with heart failure: clinical associations and prognostic value. Eur Heart J 2015, 36(12):733-742.

18. Maron BJ, Haas TS, Maron MS, Lesser JR, Browning JA, Chan RH, Olivotto I, Garberich RF, Schwartz RS: Left atrial remodeling in hypertrophic cardiomyopathy and susceptibility markers for atrial fibrillation identified by cardiovascular magnetic resonance. Am J Cardiol 2014, 113(8):1394-1400.

19. Thomas L, Abhayaratna WP: Left Atrial Reverse Remodeling: Mechanisms, Evaluation, and Clinical Significance. JACC Cardiovasc Imaging 2017, 10(1):65-77.

20. Abed HS, Wittert GA, Leong DP, Shirazi MG, Bahrami B, Middeldorp ME, Lorimer MF, Lau DH, Antic NA, Brooks AG, Abhayaratna WP, Kalman JM, Sanders P: Effect of weight reduction and cardiometabolic risk factor management on symptom burden and severity in patients with atrial fibrillation: a randomized clinical trial. JAMA 2013, 310(19):20502060.

21. Habibi M, Samiei S, Ambale Venkatesh B, Opdahl A, Helle-Valle TM, Zareian M, Almeida AL, Choi EY, Wu C, Alonso A, Heckbert SR, Bluemke DA, Lima JA: Cardiac Magnetic Resonance-Measured Left Atrial Volume and Function and Incident Atrial Fibrillation: Results From MESA (Multi-Ethnic Study of Atherosclerosis). Circ Cardiovasc Imaging 2016, 9(8):10.1161/CIRCIMAGING.115.004299.

22. Oliver W, Matthews G, Ayers CR, Garg S, Gupta S, Neeland IJ, Drazner MH, Berry JD, Matulevicius S, de Lemos JA: Factors Associated With Left Atrial Remodeling in the General Population. Circ Cardiovasc Imaging 2017, 10(2):10.1161/CIRCIMAGING.116.005047. 
23. Graca B, Ferreira MJ, Donato P, Gomes L, Castelo-Branco M, Caseiro-Alves F: Left atrial dysfunction in type 2 diabetes mellitus: insights from cardiac MRI. Eur Radiol 2014, 24(11):2669-2676.

24. Fuster JJ, Ouchi N, Gokce N, Walsh K: Obesity-Induced Changes in Adipose Tissue Microenvironment and Their Impact on Cardiovascular Disease. Circ Res 2016, 118(11):1786-1807.

25. Reingold JS, McGavock JM, Kaka S, Tillery T, Victor RG, Szczepaniak LS: Determination of triglyceride in the human myocardium by magnetic resonance spectroscopy: reproducibility and sensitivity of the method. Am J Physiol Endocrinol Metab 2005, 289(5):E935-9.

26. Goldberg IJ, Trent CM, Schulze PC: Lipid metabolism and toxicity in the heart. Cell Metab 2012, 15(6):805-812.

27. Lopaschuk GD, Folmes CD, Stanley WC: Cardiac energy metabolism in obesity. Circ Res 2007, 101(4):335-347.

28. Opacic D, van Bragt KA, Nasrallah HM, Schotten U, Verheule S: Atrial metabolism and tissue perfusion as determinants of electrical and structural remodelling in atrial fibrillation. Cardiovasc Res 2016, 109(4):527-541.

29. Sievers B, Kirchberg S, Franken U, Bakan A, Addo M, John-Puthenveettil B, Trappe HJ: Determination of normal gender-specific left atrial dimensions by cardiovascular magnetic resonance imaging. J Cardiovasc Magn Reson 2005, 7(4):677-683.

30. Maceira AM, Cosin-Sales J, Roughton M, Prasad SK, Pennell DJ: Reference left atrial dimensions and volumes by steady state free precession cardiovascular magnetic resonance. J Cardiovasc Magn Reson 2010, 12:65-429X-12-65.

31. Stojanovska J, Cronin P, Patel S, Gross BH, Oral H, Chughtai K, Kazerooni EA: Reference normal absolute and indexed values from ECG-gated MDCT: left atrial volume, function, and diameter. AJR Am J Roentgenol 2011, 197(3):631-637.

32. Maceira AM, Cosin-Sales J, Prasad SK, Pennell DJ: Characterization of left and right atrial function in healthy volunteers by cardiovascular magnetic resonance. J Cardiovasc Magn Reson 2016, 18(1):64.

33. Hudsmith LE, Cheng AS, Tyler DJ, Shirodaria C, Lee J, Petersen SE, Francis JM, Clarke K, Robson MD, Neubauer S: Assessment of left atrial volumes at 1.5 Tesla and 3 Tesla using FLASH and SSFP cine imaging. J Cardiovasc Magn Reson 2007, 9(4):673-679.

34. Le Ven F, Bibeau K, De Larochelliere E, Tizon-Marcos H, Deneault-Bissonnette S, Pibarot $P$, Deschepper $C F$, Larose $E$ : Cardiac morphology and function reference values derived from a large subset of healthy young Caucasian adults by magnetic resonance imaging. Eur Heart J Cardiovasc Imaging 2016, 17(9):981-990. 
Figure legend

Figure 1. Correlations of left atrial (LA) ejection fraction (EF) with selected contributing factors of metabolic syndrome (MetS). LA EF correlates negatively with (A) waist circumference, (B) hepatic triglyceride content (logarithmic scale), (C) visceral adipose tissue, and (D) fasting plasma glucose levels, and positively with (E) fasting serum high-density lipoprotein cholesterol (HDL). Correlation of LA EF with (F) systolic blood pressure was nonsignificant. Open circles indicate subjects with MetS and closed circles subjects without MetS. Dashed line (A) displays cut-off value $(94 \mathrm{~cm})$ of waist circumference for MetS. Abbreviations: EF, ejection fraction; LA, left atrial; HDL, high-density lipoprotein cholesterol. 

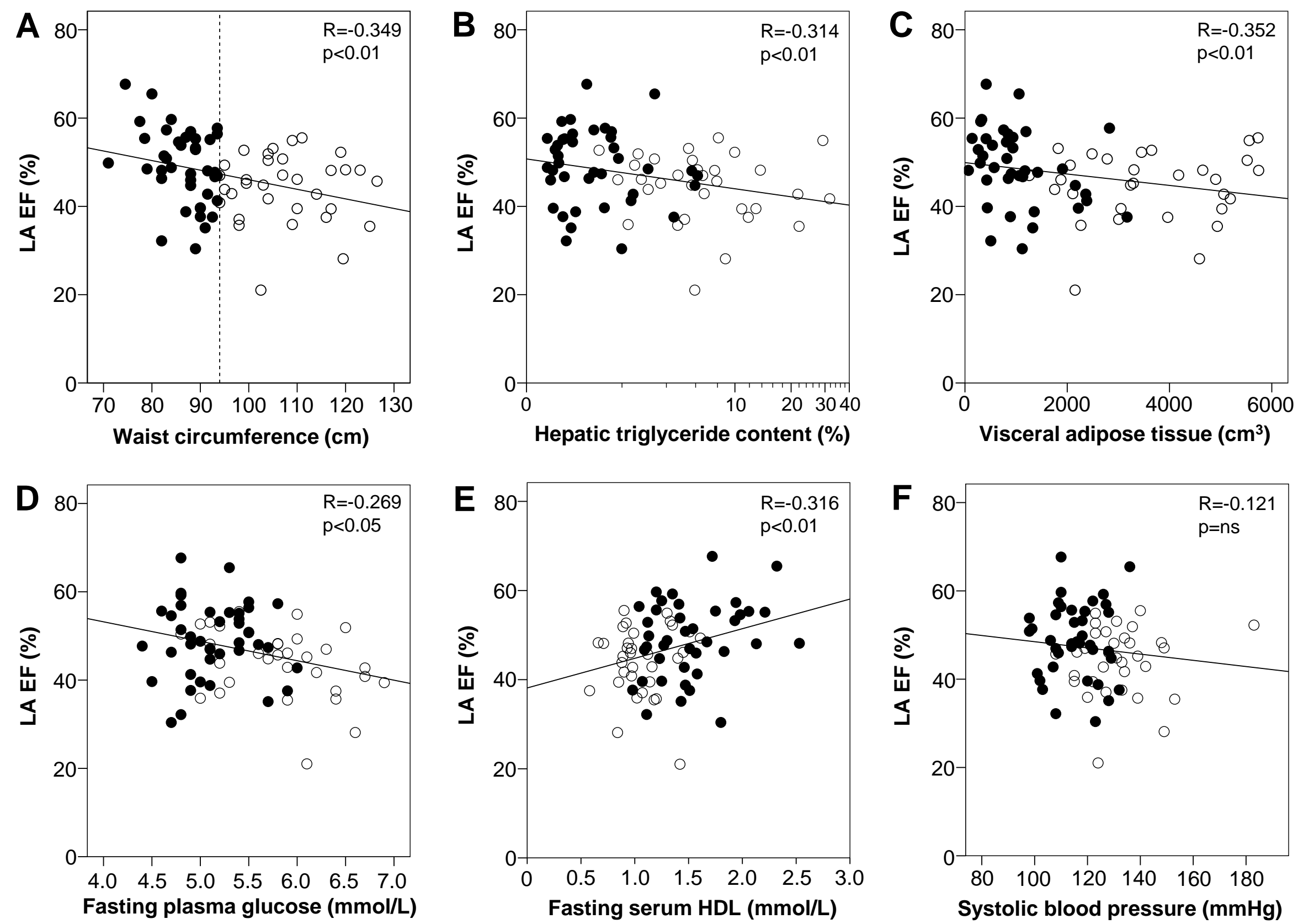


\section{Highlights}

- Metabolic syndrome associates with subclinical left atrial dysfunction.

- Multiple components of metabolic syndrome are related to left atrial dysfunction.

- Visceral fat was the best ectopic fat deposit to predict left atrial dysfunction.

- Role of cardiac steatosis in left atrial dysfunction was not essential. 